\title{
STEREOLOGY WITH CYLINDER PROBES
}

\author{
Luis M. Cruz-Orive ${ }^{\varpi, 1}$ And Ximo Gual-Arnau ${ }^{2}$
}

${ }^{1}$ Department of Mathematics, Statistics and Computation, Faculty of Sciences, University of Cantabria, Avda. Los Castros 48, E-39005 Santander, Spain, ${ }^{2}$ Department of Mathematics, Institute of New Imaging Technologies, University Jaume I, E-12071 Castelló, Spain e-mail: luis.cruz@unican.es,gual@uji.es

(Received July 6, 2020; revised August 18, 2020; accepted August 19, 2020)

\begin{abstract}
Intersection formulae of Crofton type for general geometric probes are well known in integral geometry. For the special case of cylinders with non necessarily convex directrix, however, no equivalent formula seems to exist in the literature. We derive this formula resorting to motion invariant probability elements associated with test systems, instead of using a traditional approach. Because cylinders are seldom used as probes in streological practice, however, this note is mainly of a theoretical nature.
\end{abstract}

Keywords: Cylinders, integral geometry, motion invariant measures, ratio design, stereology, test systems.

\section{INTRODUCTION}

The fundamental equations of stereology - see for instance Miles (1972), Baddeley and Jensen (2005), (Section 2.2.3), or Cruz-Orive (2017) for their history, are based on intersections between a target set and a geometric probe. The latter is usually an $r$-plane, or a bounded portion of it. With rare exceptions (e.g. Horgan et al. (1993)) cylinder probes are seldom used in stereology - therefore, the present note is mainly theoretically oriented.

The classical stereological equations are usually ratios of motion invariant measures. For instance, the ratio $B_{A}$ of the total planar curve length determined by a motion invariant test plane in the boundary of a compact three dimensional set, divided by the total planar section area determined in the set, is equal to $(\pi / 4) S_{V}$, where $S_{V}$ is the surface to volume ratio of the set. The identity is the result of dividing side by side two integral identities which belong to the family of Crofton intersection formulae of integral geometry. Such ratio identities hold formally unchanged for probes other than $r$-planes, notably cylinders. The Crofton integrals in the numerator and the denominator of a ratio, however, do in general depend on probe shape.

To fix ideas, consider a cylindrical surface $Z_{2} \subset \mathbb{R}^{3}$ whose generator $L_{1[0]}$ is a straight line and its directrix, namely its cross section by a plane $L_{2[0]}$ through the origin, perpendicular to the generator, is a piecewise smooth, simple closed curve $Z_{1} \subset L_{2[0]}$ of perimeter length $b$, see Fig. 1 . The object is a compact set $Y \subset \mathbb{R}^{3}$ of surface area $S$ and volume $V$. The motion invariant density of the cylinder is the kinematic density:

$$
\mathrm{d} Z_{2}=\mathrm{d} x \mathrm{~d} u \mathrm{~d} \tau, \quad x \in \mathbb{R}^{2}, u \in \mathbb{S}^{2}, \tau \in \mathbb{S},
$$

where $x$ is an associated point (AP) of the cross section $Z_{1}$, (namely a point rigidly attached to $Z_{1}$ according to a fixed rule), whereas $u$ is a unit vector on the unit sphere $\mathbb{S}^{2}$ giving the direction of the generator, and $\tau$ is a rotation around the generator. The pertinent Crofton intersection formulae read as follows,

$$
\begin{aligned}
& \int B\left(\partial Y \cap Z_{2}\right) \mathrm{d} Z_{2}=2 \pi^{3} b S, \\
& \int A\left(Y \cap Z_{2}\right) \mathrm{d} Z_{2}=8 \pi^{2} b V,
\end{aligned}
$$

and side by side division yields the aforementioned stereological equation $S_{V}=(4 / \pi) B_{A}$. Analogous formulae arise for a solid cylinder $Z_{3} \subset \mathbb{R}^{3}$ whose cross section $Z_{2} \subset L_{2[0]}$ is a domain of area $a$ and perimeter

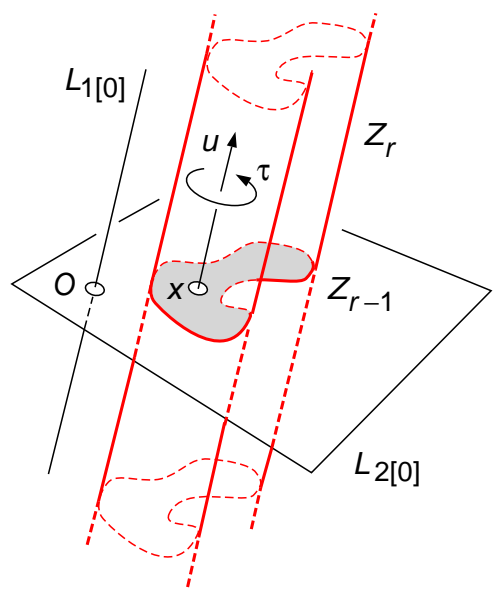

length $b$.

Fig. 1. Sketch of a cylinder $Z_{r} \subset \mathbb{R}^{3}$ with generator $L_{1[0]}$ and directrix $Z_{r-1} \subset L_{2[0]}$. For $r=1,2,3$ the cylinder is a straight line, a cylindrical surface, or a solid cylinder, respectively. The remaining symbols are defined in the text. 
While a Crofton intersection formula for general manifolds is well known, see for instance Santaló (1976), Eq. (15.20), we have not found an analogous formula for general cylinders in the literature. Schneider and Weil (2008) consider cylinders with convex directrix. Particular cases such as Eq. 2 and Eq. 3, among others, can be found in Santaló (1936), Eq. (115)-(118), who, in his Eq. (104), uses an invariant density $\mathrm{d} Z$ analogous to $\mathrm{Eq} .1$ for an unoriented cylinder $Z$, namely for $u \in \mathbb{S}_{+}^{2}$, whereby the relevant results are halved. Rey-Pastor and Santaló (1951) use the correct invariant density (Eq. (37.1)) and derive particular cases, namely Eq. (37.20), which is the same as Eq. 2 above, and Eq. (37.17) for the integral of the number of intersections between a cylinder surface and a curve in $\mathbb{R}^{3}$, see Eq. 35 below. In either of the preceding two publications the main emphasis was more on hitting measures (e.g. the measure of the number of cylinders hitting a compact set) than on intersection measures. This probably obeyed to the popularity of geometric inequalities (notably Minkowski's) over the first half of the 20th century. These preferences (at least as far as cylinders is concerned) were inherited by the books of Hadwiger (1957) and Santaló (1976). In p. 280, the latter book just reproduces the two particular formulae from Rey-Pastor and Santaló (1951) cited above.

Rey-Pastor and Santaló (1951) derive their Eq. (37.17) and Eq. (37.20) directly using ad hoc arguments which, being ingenious and elegant, lack a pattern that can be easily generalized. Here we derive a general Crofton intersection formula for cylinders (see Eq. 22 below) using invariant probability measures associated with test systems, instead of the traditional tools of integral geometry.

To make the note self contained, the main prerequisites are given next; the formula, and its proof, are given in the Results section.

\section{DEFINITIONS AND NOTATION}

\section{RELEVANT GEOMETRIC OBJECTS}

A cylinder $Z_{r}^{n} \subset \mathbb{R}^{n}$ of dimension $r \in\{1,2, \ldots, n\}$ is defined as the set product

$$
Z_{r}^{n}=L_{p[0]}^{n} \times Z_{r-p}^{n-p}, \quad p \in\{1,2, \ldots, r\},
$$

where $L_{p[0]}^{n}$ is a linear subspace of dimension $p$ through a fixed origin $O \in \mathbb{R}^{n}$, whereas $Z_{r-p}^{n-p}$ is a compact submanifold of dimension $r-p$ of class $C^{2}$, contained in the linear subspace $L_{n-p[0]}^{n}$ perpendicular to $L_{p[0]}^{n}$.

Thus, $Z_{r-p}^{n-p}$ may be visualized as: a) $Z_{r}^{n} \cap L_{n-p[0]}^{n}$, the $(r-p)$-dimensional crosssection of $Z_{r}^{n}$ determined by the $(n-p)$-plane $L_{n-p[0]}^{n}$.

b) The $(r-p)$-dimensional orthogonal projection of the cylinder $Z_{r}^{n}$ onto $L_{n-p[0]}^{n}$.

The linear subspace $L_{p[0]}^{n}$ is the generator, whereas the subset $Z_{r-p}^{n-p}$ is the directrix of the cylinder $Z_{r}^{n}$.

Examples for $n=3$ and $p=1$.

- If $r=1$, then the cylinder $Z_{1}^{3}$ is a straight line $L_{1}^{3}$ normal to a given plane $L_{2[0]}^{3}$.

- If $r=2$, then $Z_{2}^{3}$ is a cylindrical surface whose cross section is a bounded curve $Z_{1}^{2}$ contained in a plane $L_{2[0]}^{3}$ normal to the straight line generator $L_{1[0]}^{3}$.

- If $r=3$, then $Z_{3}^{3}$ is a solid cylinder whose cross section is a domain $Z_{2}^{2}$ of dimension 2 contained in $L_{2[0]}^{3}$.

An $n$-box $J_{0}^{n} \subset \mathbb{R}^{n}$ is defined as

$$
J_{0}^{n}=\left[0, a_{1}\right) \times\left[0, a_{2}\right) \times \cdots \times\left[0, a_{n}\right),
$$

where $a_{1}, a_{2}, \ldots, a_{n}$ are finite, positive real numbers.

A bounded cylinder $T_{r}^{n} \subset \mathbb{R}^{n}$ is a compact set defined as:

$$
T_{r}^{n}=Z_{r-p}^{n-p} \times J_{0}^{p} .
$$

Example. If $p=1$, then $T_{r}^{n}$ is a bounded right cylinder of base $Z_{r-1}^{n-1}$ and finite height $a_{1}>0$.

\section{MOTION INVARIANT DENSITIES}

The material in this section, given to make the note self contained, is well known - for general reference see Santaló (1976).

A non-oriented linear subspace $L_{p[0]}^{n}$ submitted to a rotation from the group $G_{p, n-p}$ of rotations about a fixed point in $\mathbb{R}^{n}$, called the Grassmann manifold, has a rotation invariant density denoted by

$$
\mathrm{d} L_{p[0]}^{n}=\mathrm{d} L_{n-p[0]}^{n} .
$$

It can be shown that

$$
\int_{G_{p, n-p}} \mathrm{~d} L_{p[0]}^{n}=\frac{O_{n-1} O_{n-2} \cdots O_{n-p}}{O_{p-1} O_{p-2} \cdots O_{0}},
$$

where

$$
O_{k}=\frac{2 \pi^{(k+1) / 2}}{\Gamma((k+1) / 2)}, \quad k=0,1, \ldots, n,
$$


denotes the surface area of the $k$-dimensional unit sphere $\mathbb{S}^{k}\left(O_{0}=2, O_{1}=2 \pi, O_{k+2}=2 \pi O_{k} /(k+1)\right)$.

As given below, the motion invariant density for cylinders involves an oriented $p$-subspace $L_{p[0]}^{n}$, which we denote by $L_{p[0]}^{*}$. Consequently, the measure given in Eq. 8 must be multiplied by $O_{0}=2$.

For a compact set $T_{r}^{n} \subset \mathbb{R}^{n}$, not necessarily a bounded cylinder, the motion invariant density is the kinematic density, namely,

$$
\mathrm{d} T_{r}^{n}=\mathrm{d} x_{n} \mathrm{~d} u_{n}, \quad x_{n} \in \mathbb{R}^{n}, u_{n} \in G_{n[0]},
$$

where $x_{n}$ is an AP fixed in $T_{r}^{n}$, whereas $u_{n}$ is an element of the special group of rotations $G_{n[0]}$, isomorphic to $S O(n)$, about a fixed point in $\mathbb{R}^{n}$. It can be shown that

$$
\int_{G_{n[0]}} \mathrm{d} u_{n}=O_{n-1} O_{n-2} \ldots O_{1} .
$$

Example. For $n=3$ we have $u_{3}=\left(u_{2}, u_{1}\right)$, where $u_{2} \equiv(\phi, \theta) \in \mathbb{S}^{2}$ is a unit vector of spherical polar coordinates $(\phi, \theta)$ whereas $u_{1} \equiv \tau \in \mathbb{S}^{1}$ is a rotation about $u_{2}$. Thus,

$$
\int_{G_{3[0]}} \mathrm{d} u_{3}=\int_{\mathbb{S}^{2}} \mathrm{~d} u_{2} \int_{\mathbb{S}^{1}} \mathrm{~d} u_{1}=4 \pi 2 \pi=8 \pi^{2} .
$$

For a cylinder $Z_{r}^{n}$ of cross section $Z_{r-p}^{n-p}$, where $1 \leq p \leq r \leq n$, the motion invariant density is

$$
\mathrm{d} Z_{r}^{n}=\mathrm{d} Z_{r-p}^{n-p} \mathrm{~d} L_{p[0]}^{*},
$$

(Santaló, 1976, Eq. (15.76)), where $\mathrm{d} Z_{r-p}^{n-p}$ is the kinematic density in $L_{n-p[0]}$, namely,

$$
\mathrm{d} Z_{r-p}^{n-p}=\mathrm{d} x_{n-p} \mathrm{~d} u_{n-p} .
$$

Substitution into the right hand side of Eq. 13 yields,

$$
\mathrm{d} Z_{r}^{n}=\mathrm{d} x_{n-p} \mathrm{~d} L_{p[0]}^{*} \mathrm{~d} u_{n-p},
$$

where $x_{n-p} \in L_{n-p[0]}^{n}, \quad L_{p[0]}^{*} \in G_{p, n-p}^{*}, \quad u_{n-p} \in$ $G_{n-p[0]}$ (this group is isomorphic to the group of rotations about $\left.L_{p[0]}^{*}\right)$. From Eq. 8 and Eq. 11 we get,

$$
\int_{G_{p, n-p}^{*}} \mathrm{~d} L_{p[0]}^{*} \int_{G_{n-p[0]}} \mathrm{d} u_{n-p}=O_{n-1} \ldots O_{p}
$$

Example. For a cylinder $Z_{r}^{3}, r=2,3, p=1$, the motion invariant density given by Eq. 15 reduces to Eq. 1 .

\section{TEST SYSTEMS OF CYLINDERS}

Consider a test system $\Lambda_{T} \subset \mathbb{R}^{n}$ whose fundamental tile is an $n$-box $J_{0}^{n}$, whereas the fundamental probe is a bounded cylinder $T_{r}^{n} \subset J_{0}^{n}$ given by Eq. 6 with its $\mathrm{AP}$ at the origin $O$, see Fig. 2. For details pertaining to the construction of a test system see Santaló (1976) under the term "lattice of figures".

(a)
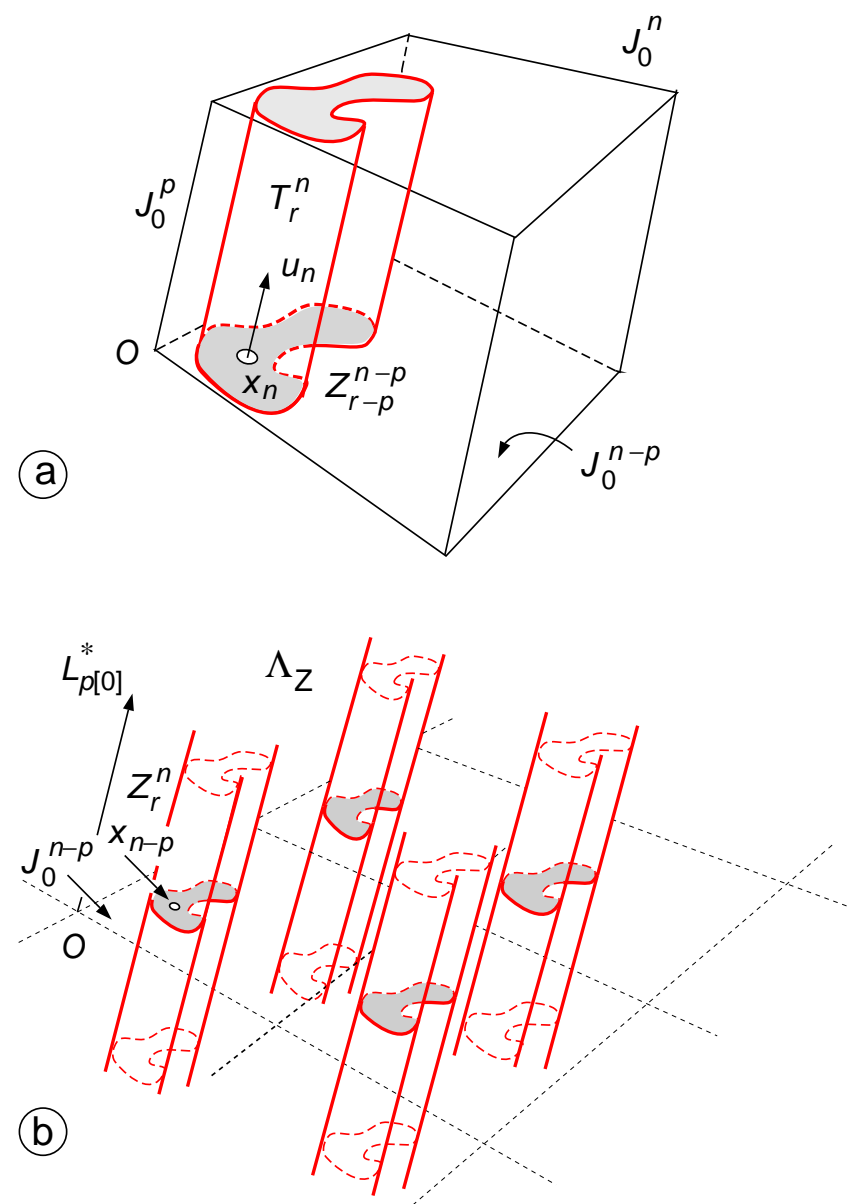

Fig. 2. (a) Fundamental tile ( $n-b o x) J_{0}^{n}$ of a test system $\Lambda_{T}$. The fundamental probe $T_{r}^{n} \subset J_{0}^{n}$ is a bounded cylinder given by Eq. 6. (b) A portion of the test system $\Lambda_{T}$ generated by $T_{r}^{n}$, see $(a)$, which is congruent with a test system $\Lambda_{Z}$ of cylinders.

The motion invariant probability element adopted for $\Lambda_{T}$, for which we use the shorthand notation $\mathbb{P}\left(\mathrm{d} \Lambda_{T}\right)$, is the normalitzed version of the kinematic density $\mathrm{d} T_{r}^{n}$, as follows. For $x_{n} \in J_{0}^{n}, u_{n} \in G_{n[0]}$,

$$
\mathbb{P}\left(\mathrm{d} \Lambda_{T}\right) \equiv \mathbb{P}\left(\mathrm{d} x_{n}, \mathrm{~d} u_{n}\right)=\frac{\mathrm{d} x_{n}}{v_{n}\left(J_{0}^{n}\right)} \frac{\mathrm{d} u_{n}}{\int \mathrm{d} u_{n}},
$$

where $v_{d}(\cdot)$ denotes $d$-Hausdorff measure. The integral is given by Eq. 11. For details see Cruz-Orive (2002), or Appendix A in Voss and Cruz-Orive (2009).

Because $T_{r}^{n}=Z_{r-p}^{n-p} \times J_{0}^{p}, J_{0}^{n}=J_{0}^{n-p} \times J_{0}^{p}$, and $Z_{r-p}^{n-p} \subset J_{0}^{n-p}$, any stack of probes of $\Lambda_{T}$ is in fact 
an infinite cylinder $Z_{r}^{n}$. Thus, $\Lambda_{T}$ coincides with a test system $\Lambda_{Z} \subset \mathbb{R}^{n}$ of cylinders congruent with $Z_{r}^{n}$, whose fundamental tile is $J_{0}^{n-p} \subset L_{n-p[0]}^{n}$, whereas the fundamental probe is the orthogonal projection $Z_{r-p}^{n-p}$ of $Z_{r}^{n}$ onto $L_{n-p[0]}^{n}$. The motion invariant probability element corresponding to $\Lambda_{Z}$ is the normalized density given by Eq. 15. Thus, for $x_{n-p} \in J_{0}^{n-p}, L_{p[0]}^{*} \in$ $G_{p, n-p}^{*}, u_{n-p} \in G_{n-p[0]}$,

$$
\begin{aligned}
\mathbb{P}\left(\mathrm{d} \Lambda_{Z}\right) & \equiv \mathbb{P}\left(\mathrm{d} x_{n-p}, \mathrm{~d} L_{p[0]}^{*}, \mathrm{~d} u_{n-p}\right) \\
& =\frac{\mathrm{d} x_{n-p}}{v_{n-p}\left(J_{0}^{n-p}\right)} \frac{\mathrm{d} L_{p[0]}^{*} \mathrm{~d} u_{n-p}}{\int \mathrm{d} L_{p[0]}^{*} \mathrm{~d} u_{n-p}}
\end{aligned}
$$

where the integral involving the orientation variables is given by Eq. 16 .

From the preceding considerations it follows that the test system $\Lambda_{Z}$ equipped with the probability element given by Eq. 18, has identical statistical properties as the test system $\Lambda_{T}$ equipped with the probability element given by Eq. 17. In particular, for a compact submanifold $Y_{q} \subset \mathbb{R}^{n}$ of dimension $q \in\{0,1, \ldots, n\}$, with $q+r \geq n$, the following identity holds,

$$
\mathbb{E} v_{q+r-n}\left(Y_{q} \cap \Lambda_{T}\right)=\mathbb{E} v_{q+r-n}\left(Y_{q} \cap \Lambda_{Z}\right),
$$

the expectations being with respect to the corresponding motion invariant probability elements.

\section{CROFTON INTERSECTION FORMULA FOR BOUNDED PROBES}

For the compact submanifold $Y_{q} \subset \mathbb{R}^{n}$ just considered, hit by a compact probe $T_{r}^{n}$ equipped with the kinematic density $\mathrm{d} T_{r}^{n}$ given by Eq. 10 , the following identity holds,

$$
\begin{array}{r}
\int_{\mathbb{R}^{n} \times G_{n[0]}} v_{q+r-n}\left(Y_{q} \cap T_{r}^{n}\right) \mathrm{d} T_{r}^{n} \\
=c(q, r, n) v_{q}\left(Y_{q}\right) v_{r}\left(T_{r}^{n}\right),
\end{array}
$$

(Santaló (1976), Eq. (15.20)), where

$$
c(q, r, n)=\frac{O_{n} O_{n-1} \cdots O_{1} O_{q+r-n}}{O_{q} O_{r}} .
$$

\section{RESULTS}

\section{CROFTON INTERSECTION FORMULA FOR CYLINDERS}

The main purpose of this note is to prove the following identity.

\section{Proposition}

$$
\begin{gathered}
\int_{\mathbb{R}^{n-p} \times G_{p, n-p}^{*} \times G_{n-p[0]}} v_{q+r-n}\left(Y_{q} \cap Z_{r}^{n}\right) \mathrm{d} Z_{r}^{n} \\
=c_{Z}(q, r, n, p) v_{r-p}\left(Z_{r-p}^{n-p}\right) v_{q}\left(Y_{q}\right)
\end{gathered}
$$

where

$$
c_{Z}(q, r, n, p)=\frac{O_{n} O_{n-1} \cdots O_{p} O_{q+r-n}}{O_{q} O_{r}} .
$$

\section{Proof}

Set $v \equiv v_{q+r-n}, Y \equiv Y_{q}, T \equiv T_{r}^{n}, Z \equiv Z_{r}^{n}, Z^{\prime} \equiv Z_{r-p}^{n-p}$ and $c \equiv c(q, r, n)$, for short. In addition, the domains of integration $G_{n[0]}$ for $u_{n}, G_{p, n-p}^{*}$ for $L_{p[0]}^{*}$, and $G_{n-p[0]}$ for $u_{n-p}$, will be omitted in the sequel.

The proof is based on Eq. 19, whose left hand side becomes,

$$
\begin{aligned}
& \mathbb{E} v\left(Y \cap \Lambda_{T}\right) \\
& =\int_{x_{n} \in J_{0}^{n}} v\left(Y \cap \Lambda_{T}\right) \mathbb{P}\left(\mathrm{d} \Lambda_{T}\right) \\
& =\frac{1}{v_{n}\left(J_{0}^{n}\right) \int \mathrm{d} u_{n}} \int_{x_{n} \in J_{0}^{n}} v\left(Y \cap \Lambda_{T}\right) \mathrm{d} x_{n} \mathrm{~d} u_{n} \\
& =\frac{1}{v_{n}\left(J_{0}^{n}\right) \int \mathrm{d} u_{n}} \int_{x_{n} \in \mathbb{R}^{n}} v(Y \cap T) \mathrm{d} T,
\end{aligned}
$$

where the last identity follows from Santaló's identity for test systems, see Santaló (1976), chapter 8, or CruzOrive (2002). In combination with Eq. 20 we obtain,

$$
\mathbb{E} v\left(Y \cap \Lambda_{T}\right)=\frac{c v_{q}(Y) v_{r}(T)}{v_{n}\left(J_{0}^{n}\right) \int \mathrm{d} u_{n}} .
$$

Analogously,

$$
\begin{aligned}
& \mathbb{E} v\left(Y \cap \Lambda_{Z}\right) \\
& =\int_{x_{n-p} \in J_{0}^{n-p}} v\left(Y \cap \Lambda_{Z}\right) \mathbb{P}\left(\mathrm{d} \Lambda_{Z}\right) \\
& =\frac{\int_{x_{n-p} \in \mathbb{R}^{n-p}} v(Y \cap Z) \mathrm{d} Z}{v_{n-p}\left(J_{0}^{n-p}\right) \int d L_{p[0]}^{*} \mathrm{~d} u_{n-p}} .
\end{aligned}
$$

Finally, bearing Eq. 11 and Eq. 16 in mind, applying Eq. 19, and using the following identities,

$$
\begin{gathered}
v_{n}\left(J_{0}^{n}\right)=v_{n-p}\left(J_{0}^{n-p}\right) v_{p}\left(J_{0}^{p}\right), \\
v_{r}(T)=v_{r-p}\left(Z^{\prime}\right) v_{p}\left(J_{0}^{p}\right),
\end{gathered}
$$

we obtain,

$$
\begin{aligned}
& \int_{x_{n-p} \in \mathbb{R}^{n-p}} v(Y \cap Z) \mathrm{d} Z \\
& =\frac{O_{n} O_{n-1} \cdots O_{p} O_{q+r-n}}{O_{q} O_{r}} v_{r-p}\left(Z^{\prime}\right) v_{q}(Y) .
\end{aligned}
$$


which is the identity given by Eq. 22, thus completing the proof of the proposition.

\section{SPECIAL CASES FOR $n=2,3$ AND $p=1$}

A cylinder $Z_{2}^{2} \subset \mathbb{R}^{2}$ is a solid stripe of thickness $t>0$, say, in the plane. Its boundary $\partial Z_{2}^{2} \equiv Z_{1}^{2}$ is the union of two parallel straight lines a distance $t$ apart.

Consider a compact set $Y_{2} \subset \mathbb{R}^{2}$ of area $A>0$ and piecewise smooth boundary $\partial Y_{2} \equiv Y_{1}$ of length $B>0$. Application of Eq. 22 with $r=1$ yields:

$$
\int v_{0}\left(Y_{1} \cap Z_{1}^{2}\right) \mathrm{d} Z_{1}^{2}=\frac{O_{2} O_{1} O_{0}}{O_{1} O_{1}} v_{0}\left(Z_{0}^{1}\right) B=8 B,
$$

because the projection $Z_{0}^{1}$ of $Z_{1}^{2}$ onto an axis normal to the stripe is the union of two points a distance $t$ apart hence $v_{0}\left(Z_{0}^{1}\right)=2$. As a cross-check, note that

$$
\begin{aligned}
\int v_{0}\left(Y_{1} \cap Z_{1}^{2}\right) \mathrm{d} Z_{1}^{2} & =2 \int I\left(Y_{1} \cap L_{1}^{*}\right) \mathrm{d} L_{1}^{*} \\
& =4 \int I\left(Y_{1} \cap L_{1}^{2}\right) \mathrm{d} L_{1}^{2}=8 B,
\end{aligned}
$$

where $L_{1}^{2}$ is a straight line with motion invariant density $\mathrm{d} L_{1}^{2}$, and $I(\cdot)$ denotes number of intersections - see Cruz-Orive (2017), Eq. (1), for references.

On the other hand

$$
\int v_{2}\left(Y_{2} \cap Z_{2}^{2}\right) \mathrm{d} Z_{2}^{2}=\frac{O_{2} O_{1} O_{2}}{O_{2} O_{2}} v_{1}\left(Z_{1}^{1}\right) A=2 \pi t A,
$$

which is twice the value obtained in the classical manner (see Eq. (5.16) of Santaló (1976)), because we consider oriented stripes. Note that the projection $Z_{1}^{1}$ of the stripe $Z_{2}^{2}$ onto an axis normal to the stripe is a segment of length $t$ - hence $v_{1}\left(Z_{1}^{1}\right)=t$.

Further

$$
\int v_{1}\left(Y_{2} \cap Z_{1}^{2}\right) \mathrm{d} Z_{1}^{2}=\frac{O_{2} O_{1} O_{1}}{O_{2} O_{1}} v_{0}\left(Z_{0}^{1}\right) A=4 \pi A,
$$

which is equivalent to

$$
\int v_{1}\left(Y_{2} \cap Z_{1}^{2}\right) \mathrm{d} Z_{1}^{2}=4 \int L\left(Y_{2} \cap L_{1}^{2}\right) \mathrm{d} L_{1}^{2}=4 \pi A,
$$

where $L(\cdot)$ denotes intercept length, and the first integral pertains to the two oriented straight lines constituting $Z_{1}^{2}$, yielding $2 \pi A$ each.

For $n=3$ and $r=q=2$, Eq. 22 yields $c_{Z}(2,2,3,1)=$ $\mathrm{O}_{3} \mathrm{O}_{2} \mathrm{O}_{1} \mathrm{O}_{1} /\left(\mathrm{O}_{2} \mathrm{O}_{2}\right)=2 \pi^{3}$, and we obtain Eq. 2 .

For $n=3, q=3$, and $r=2$ Eq. 22 yields $c_{Z}(3,2,3,1)=\mathrm{O}_{3} \mathrm{O}_{2} \mathrm{O}_{1} \mathrm{O}_{2} /\left(\mathrm{O}_{3} \mathrm{O}_{2}\right)=8 \pi^{2}$ and we obtain Eq. 3 .
Finally, for $n=3, r=2$, and $q=1$, namely for $Z_{2}^{3} \subset \mathbb{R}^{3}$ hitting a curve $Y_{1} \subset \mathbb{R}^{3}$ of length $L$, we have $c_{Z}(1,2,3,1)=O_{3} O_{0}=4 \pi^{2}$ and,

$$
\int v_{0}\left(Y_{1} \cap Z_{2}^{3}\right) \mathrm{d} Z_{2}^{3}=4 \pi^{2} b L
$$

see Santaló (1976), p. 280.

\section{STEREOLOGICAL EQUATIONS FOR TEST SYSTEMS OF CYLINDERS}

Substitution of Eq. 29 into the right hand side of Eq. 26 , yields the Hausdorff measure $v_{q}\left(Y_{q}\right)$ of a compact submanifold $Y_{q} \subset \mathbb{R}^{n}$ in terms of the measure of its intersection with a test system $\Lambda_{Z_{r}}$ of cylinders of dimension $r$, namely,

$v_{q}\left(Y_{q}\right)=\frac{O_{q} O_{r}}{O_{n} O_{q+r-n}} \frac{v_{n-p}\left(J_{0}^{n-p}\right)}{v_{r-p}\left(Z_{r-p}^{n-p}\right)} \cdot \mathbb{E} v_{q+r-n}\left(Y_{q} \cap \Lambda_{Z_{r}}\right)$.

The numerical constant in the right hand side of the preceding identity is the same as that arising for $r$-probes in general, see for instance Voss and CruzOrive (2009), Eq. (A28), (A32).

In stereology, $v_{q}\left(Y_{q}\right)$ is often estimated via the ratio design, which is based on the identity,

$$
v_{q}\left(Y_{q}\right)=v_{n}\left(X_{n}\right) \cdot R_{q, n},
$$

where $X_{n} \supset Y_{q}$ is a reference submanifold containing $Y_{q}$, whose volume is estimated separately (e.g. by the Cavalieri design). Ratio designs were studied in some detail by Cruz-Orive (1980) and Cruz-Orive and Weibel (1981), see also Baddeley and Jensen (2005). Thus, it only remains to estimate the ratio $R_{q, n} \equiv v_{q}\left(Y_{q}\right) / v_{n}\left(X_{n}\right)$ via the identity,

$$
R_{q, n}=\frac{O_{q} O_{r}}{O_{n} O_{q+r-n}} \cdot \frac{\mathbb{E} v_{q+r-n}\left(Y_{q} \cap \Lambda_{Z_{r}}\right)}{\mathbb{E} v_{r}\left(X_{n} \cap \Lambda_{Z_{r}}\right)} .
$$

Provided that the same test system $\Lambda_{Z_{r}}$ is used in the numerator and denominator, the right hand side of the preceding identity does not involve any properties of $\Lambda_{Z_{r}}$ itself - hence the relative popularity of ratios. Thus, with the usual conditions Eq. 38 holds for any $r$-dimensional test system; it was already obtained by Miles (1972), Eq. (2.16), and it encapsulates the classical stereological equations used in practice, see also Cruz-Orive (2002), Eq. (6.19).

\section{ACKNOWLEDGMENTS}

Work supported by Project UJIB2017-13 from Universitat Jaume I, Spain, and by Grant DPI201787333-R from the Spanish Ministry of Science, Innovation and Universities. 


\section{REFERENCES}

Baddeley A, Jensen EBV (2005). Stereology for statisticians. Boca Raton: Chapman and Hall/CRC.

Cruz-Orive LM (1980). Best linear unbiased estimators for stereology. Biometrics 36:595-605.

Cruz-Orive LM (2002). Stereology: meeting point of integral geometry, probability, and statistics. In memory of Professor Luis A.Santaló (1911-2001). Special issue (Homenaje a Santaló), Mathematicae Notae 41:49-98.

Cruz-Orive LM (2017). Stereology: A historical survey. Image Anal Stereol 36:156-177.

Cruz-Orive LM, Weibel E (1981). Sampling designs for stereology. J Microsc 122:235-72.

Hadwiger H (1957) Vorlesungen über Inhalt, Oberfläche und Isoperimetrie. Berlin: Springer.
Horgan GW, Buckland ST, Mackie-Dawson LA (1993). Estimating three-dimensional line process densities from tube counts. Biometrics 49:899-906.

Miles RE (1972). Multidimensional perspectives in stereology. J Microsc 95:181-95.

Rey-Pastor J, Santaló LA (1951). Geometría Integral. Madrid: Espasa-Calpe.

Santaló LA (1936) Integralgeometrie 5. Über das kinematische Mass im Raum. Paris: Hermann.

Santaló LA (1976). Integral Geometry and Geometric Probability. London: Addison-Wesley Publishing Company Inc.

Schneider R, Weil W (2008). Stochastic and Integral Geometry. Berlin:Springer.

Voss F, Cruz-Orive LM (2009). Second moment formulae for geometric sampling with test probes. Statistics 43:329-65. 